\title{
Help! Our Tinnitus Patients Want A Drug?
}

\author{
Mohamed Salah Elgandy ${ }^{1,2 *}$, Richard Tyler $^{2,3}$ and Claudia Coelho ${ }^{2,4}$ \\ ${ }^{1}$ Department of Otolaryngology Head and Neck Surgery, Zagazig University, Egypt \\ ${ }^{2}$ Department of Otolaryngology Head and Neck Surgery, University of Iowa, Iowa City, USA \\ ${ }^{3}$ Department of Communication Sciences and Disorders, University of Iowa, Iowa City, USA \\ ${ }^{4}$ Department of Otolaryngology, Univates Medical School, Brazil
}

*Corresponding author: Mohamed Salah Elgandy, Department of Otolaryngology Head and Neck Surgery, Zagazig University, Egypt

\begin{abstract}
Tinnitus is common and can severely affect life quality in many. There are no FDA approved drugs for tinnitus treatment and no surgery for sensorineural tinnitus. Up till now, there are no medications or supplements approved for tinnitus; our impression is that they are widely used. Therefore, we believe it is helpful to review the literature and provide some suggestions for clinicians and sufferers. We review studies which describe current and emerging pharmacotherapies. In addition, we describe recent advances in the tinnitus field which may help overcome obstacles faced in the pharmacological treatment of tinnitus.
\end{abstract}

\section{Introduction}

Tinnitus is a common complaint and can be very debilitating [1]. The prevalence of tinnitus in adult populations ranges from $7 \%$ to $19 \%$. It increases with age [2]. In up to $5 \%$ of the adult population, tinnitus interferes negatively with the ability to lead a normal daily life; affecting many activities of daily life; including (a) thought and emotions, (b) hearing, (c) sleep, and (d) concentration. Each person is affected differently [3]. Several counseling and sound therapies are used and are helpful to many patients Tyler [4]. The Psychological Model of tinnitus considered as an important distinction between the tinnitus from reactions to the tinnitus [5]. The counseling and sound therapy help with the reactions, but tinnitus suffers often prefer pill [6]. Medications can be used to treat patients with depression and anxiety and to help with sleep problems, but do not directly change the tinnitus. Despite the significant clinical need for effective treatment of tinnitus, there is currently no single U.S Food and Drug (FDA)-approved medication for treatment of tinnitus. Various drug regimens have been tried and a few of them had shown some favorable results, but most did not result in significant benefits [7]. As with any bothersome, common disorder which lacks understanding and effective treatments, tinnitus considered as an easy target for scams [8]. Thousands of purported cures can be found on the internet and in stores providing 'over the counter' pills. People waste billions of dollars on tinnitus treatment yearly. Some scams are blatant. Others are subtle.

\section{Drugs for Tinnitus Treatment}

It seems unlikely that there will be one single drug which will cure all forms of tinnitus. It is likely that many subtypes of tinnitus exist and that each will require a different form of treatment. Thus, one of the pressing needs in tinnitus research is a scheme to classify patients into subtypes that might respond positively to a specific drug treatment. Indeed, some drugs have been reported to provide some kind of symptoms relief in a subset of patients but in others not [9]. We will review some of these drugs which might help in tinnitus symptoms relief.

\section{Anti-Arrhythmic}

\section{a) Lidocaine}

In 1935 , lidocaine was suggested and used to suppress tinnitus through nasal administration and since then many clinical studies confirmed transient suppression of tinnitus through lidocaine administration [10]. Its effect seems to be dose dependent with suppression of tinnitus occurring at free arterial plasma concentrations from 1.75 to $3.5 \mu \mathrm{mol} / \mathrm{L}$. However, it was found that concentrations more than $3.5 \mu \mathrm{mol} / \mathrm{L}$ might induce tinnitus [11]. The mechanism of action by which intravenous lidocaine might suppresses tinnitus is incompletely understood, but there is evidence that it affects both the cochlea and the central nervous 
system [7]. It is used as local anesthetic and anti-arrhythmic, it acts mainly through binding to fast voltage-gated sodium channels, reducing the magnitude of the sodium current during depolarization [12]. It also affects calcium, potassium, and glycine-evoked chloride currents [13]. Since the effects of lidocaine are short lived and due to its potentially life-threatening side effects, like cardiac arrhythmia, drowsiness, dizziness, confusion, and restlessness; lidocaine is not an option for long-term treatment. Oral analogues of lidocaine such as tocainide have been evaluated as a potential long-term therapy for tinnitus. However, several randomized, controlled studies found that tocainide had little benefit for tinnitus [14]. A nonsterile patch (Lido PAIN TV, EPI Cept) has been developed for delivery of lidocaine applied over preauricular skin. Its clinical efficacy is under clinical trial. It is produced under the pharmaceutical company EPI Cept which has less systemic concentrations than intravenous lidocaine which is needed to suppress tinnitus [5].

\section{Anti-Depressants}

The tricyclic antidepressants nortriptyline, amitriptyline and trimipramine have been investigated for the treatment of tinnitus. Beside their antidepressant properties, tricyclics have been shown to be highly efficient for the treatment of chronic pain, which is of interest in view of the proposed etiological similarities between tinnitus and neuropathic pain [16]. Examples of tricyclic antidepressant which can be used for tinnitus include (amitriptyline, trimipramine, and nortriptyline), the latter found to inhibits mainly the reuptake of norepinephrine and to a lesser extent serotonin, whereas amitriptyline inhibits the reuptake of serotonin and noradrenaline almost equally, on the other hand trimipramine differs in its mode of action from other tricyclic antidepressants in that it blocks postsynaptic dopamine and serotonin receptors [17]. From all tricyclic antidepressants, nortriptyline is worth noting. Sullivan et al. [18] reported smallscale, single blind placebo-washout study involving patients with severe tinnitus and major depression, nortriptyline significantly reduced depression and tinnitus loudness (10 $\mathrm{dB}$ reduction) in some. In a follow-up double blind placebo- controlled study involving subjects with severe tinnitus and severe depression or depressive symptoms, nortriptyline significantly reduced depression scores, tinnitus disability scores, and tinnitus loudness (6.4 $\mathrm{dB}$ reduction) relative to a placebo [19]. They concluded that there was a significant correlation between the reduction in tinnitus disability scores and depression scores, suggesting that nortriptyline is effective in reducing tinnitus loudness and severity in severely depressed tinnitus patients. There was less benefit in non-depressed individuals [20].

\section{a) Amitriptyline}

Bayaretal. [21] reported a study $(n=37)$ to compare amitriptyline with placebo and found after 6 weeks of $100 \mathrm{mg}$ amitriptyline, a significant reduction of tinnitus complaints and tinnitus loudness compared to the placebo group. Podoshin et al. [22] reported another study $(n=225)$, where amitriptyline was compared with biofeedback, and $27.5 \%$ of patients reported improvement with amitriptyline while $43.5 \%$ reported improvement with biofeedback revealing the superiority of biofeedback.

\section{b) Trimipramine}

Mihail et al. [23] evaluated Trimipramine small double-blind placebo cross-over study $(n=19)$ and did not demonstrate any difference between trimipramine and placebo treatment.

\section{c) Selective serotonin reuptake inhibitors (SSRI)}

SSRI such as sertraline have been tested. In a randomized double-blind placebo-controlled study $(n=76)$, patients without severe hearing loss, but with depression, anxiety, and a high risk for developing severe tinnitus, sertraline was significantly more effective than placebo in reducing tinnitus loudness and tinnitus severity [24].

On the other hand, paroxetine was evaluated in a doubleblind, placebo-controlled study involving chronic tinnitus patients without comorbid depression, the paroxetine group showed little difference from placebo on tinnitus loudness, Tinnitus Handicap Questionnaire (THQ) scores, and other measures [25]. Collectively, the results suggest that tinnitus patients with depression and anxiety may benefit from antidepressants [25]. However, this effect may not to be a direct effect of antidepressants on tinnitus severity, but rather due to the beneficial effect of antidepressants on comorbid depression and anxiety. Dose of antidepressants for the treatment of tinnitus is in a similar range as that used in the treatment of depression. In general, a low starting dose and slow increase of the dosage reduce side effects. Since beneficial effects do not occur immediately, minimum treatment duration of 6-12 weeks at the effective dose is recommended. If treatment effects are unsatisfactory and the decision is made to discontinue or change treatment, dosage should be reduced slowly. If a patient experiences beneficial effects, treatment should be continued at a stable effective dose for about 6 months, then the dose can be reduced over the course of weeks to months. Should the tinnitus get worse during a reduction of the dose, it is recommended to keep the dosage at the minimum providing relief.

\section{Anxiolytics}

Since tinnitus is thought to be the result of an imbalance between excitatory and inhibitory neurotransmission toward the GABA receptor [26], and benzodiazepines are allosteric potentiators of the GABAA receptor so, they have a positive effect on tinnitus by increasing inhibitory neurotransmission. Furthermore, due to their anxiolytic and sleep-inducing properties, benzodiazepines should have beneficial effects on comorbid anxiety and insomnia, and thus may help patients cope with their tinnitus.

\section{a) Alprazolam}

Johnson et al. [27] reported double-blind placebo-controlled study(n=36), 12 weeks of alprazolam administration at an individually adjusted dosage reduced tinnitus loudness in $76 \%$ of subjects measured with a tinnitus synthesizer and a visual analog scale - whereas only $5 \%$ showed a reduction in tinnitus loudness in the control group. Although the strong positive effects of alprazolam are encouraging, the study has been criticized because of the small sample size, drug dosing method, and failure to assess emotional effect. 


\section{b) Clonazepam}

Bahmad et al. [28] Stated in their retrospective study of medical records from over 30patients taking clonazepam $(0.5-1 \mathrm{mg} /$ day, 60-180 days) for vestibular or cochleovestibular disorders, that $32 \%$ of patients reported an improvement in their tinnitus. Due to their immediate effects, short-acting benzodiazepines such as lorazepam or alprazolam are widely used for acute treatment of anxiety, agitation, and insomnia, symptoms that frequently occur with tinnitus. The longer acting clonazepam provides some relief in a considerable subgroup of patients. The use of benzodiazepines should be restricted to short periods of time due to the risk of drug dependency. Moreover, caution is warranted since protracted tinnitus has been reported after discontinuation of benzodiazepines [29].

\section{Anticonvulsants}

Anticonvulsants are increasingly used in the treatment of several non-epileptic conditions, including various psychiatric disorders and pain syndromes [30]. Some of them have also been investigated for the treatment of tinnitus. As this anticonvulsant act on voltage gated Sodium and calcium channels, and on synaptic transmission mainly mediated by gamma amino butyric acid type A (GABAA) receptors [31] with resultant reduction in neuronal excitability, thus they should be beneficial for the treatment of tinnitus.

\section{a) Carbamazepine}

Carbamazepine binds to voltage-gated sodium channels and stabilizes the sodium inactivation state, thereby reducing neural firing [32], it had been investigated for tinnitus and results were mixed. Based on the assumption that carbamazepine resembles lidocaine in its mechanism of action, studies investigated the effect of carbamazepine in tinnitus patients who previously had responded to intravenous lidocaine [33]. About half of these patients had a positive response to carbamazepine (600-1,000 mg daily). A significant benefit from carbamazepine has been reported for a rare group of patients who have intermittent tinnitus that sounds like a typewriter, popcorn, or ear clicking, and which is caused by a neurovascular conflict [34].

\section{b) Gabapentin}

The anticonvulsant gabapentin acts on voltage gated calcium channels and is also used for the treatment of seizures, neuropathic pain, and migraine [35]. Results with gabapentin for the treatment of tinnitus are contradictory; one controlled trial $(n=39)$ has shown a significant improvement in tinnitus annoyance and loudness for a subgroup of participants with tinnitus related to acoustic trauma [36], other study ( $n=76$ ) did not detect any improvement in tinnitus handicap, but did report a significant improvement in tinnitus annoyance when compared to placebo [37].

\section{Anti-Glutamatergic Agents}

Glutamate receptor antagonists have been tried in tinnitus sufferers. The rationale behind it is that imbalance between inhibitory versus excitatory neurotransmission which was observed in several regions of the auditory pathway in tinnitus development thus blocking glutamatergic neurotransmission could also exert neuro protectant effects [38].

\section{a) Caroverine}

Caroverine, a spasmolytic drug, which is an antagonist of non$\mathrm{N}$-methyl-D-aspartate (NMDA) and NMDA glutamate receptors [39], has been investigated in patients with putative cochlear tinnitus. Patients were randomized to receive either caroverine (I.V., maximum dose $160 \mathrm{mg}$ ) or placebo. Within the caroverine group, $63 \%$ showed a reduction in loudness immediately after treatment and $43 \%$ still showed improvement one week later, whereas there was no improvement in the placebo group [40].

\section{b) Acamprosate}

Acamprosate is non-selective NMDA receptor which had been tried in a double-blind study $(n=50)$, Patients received placebo or acamprosate (333 mg, three times per day) and rated the loudness and annoyance of their tinnitus before and at monthly intervals of treatment. Acamprosate had no beneficial effects after 30 days of treatment, a modest benefit at 60 days, and a significant effect at 90 days. Approximately $87 \%$ of the subjects in the acamprosate group showed some improvement, including three subjects in which tinnitus disappeared, compared to $44 \%$ in the placebo group [41]. This study has not been published but the main author said it had no effect on tinnitus. A larger clinical trial is currently underway to analyze the encouraging results from this preliminary study (http://clinicaltrials.gov/ct2/show/NCT00596531).

\section{Dopaminergic-Antidopaminergic Drugs}

Dopaminergic pathways in limbic and prefrontal areas found to be involved in mediating emotional aspects of tinnitus so both dopaminergic and antidopaminergic drugs have been proposed for treating tinnitus.

\section{a) Sulpiride}

An antipsychotic drug that selectively blocks dopamineD2 receptors [42], significantly reduced subjective ratings of tinnitus and tinnitus visual analogue scores in one double-blind, placebocontrolled study. Further studies investigated combinations of sulpiride with melatonin, A prospective, randomized, doubleblinded, placebo-controlled study was done $(n=120)$ patients consulted for subjective tinnitus. They were included randomly in four groups of 30 . One group took sulpiride $(50 \mathrm{mg} / 8 \mathrm{~h})$ alone, the second group took melatonin $(3 \mathrm{mg} / 24 \mathrm{~h})$, the third group took the same doses of sulpiride $(50 \mathrm{mg} / 8 \mathrm{~h})$ plus melatonin $(3$ $\mathrm{mg} / 24 \mathrm{~h}$ ), and the fourth group took placebo (lactose $50 \mathrm{mg} / 8$ h), all for 1 month, Subjective grading of tinnitus perception and a visual analogue scale (0-10) were done for evaluation of results. Based on the subjective grading, tinnitus perception diminished by $56 \%$ in patients treated with sulpiride, by $40 \%$ in patients treated with melatonin, by $81 \%$ in patients treated with sulpiride plus melatonin, and by $22 \%$ in patients treated with placebo, concluding that Sulpiride and melatonin reduce tinnitus perception, decreasing dopamine activity. The tinnitus auditor-limbic dopaminergic pathway has broad therapeutic implications [43]. 


\section{b) Piribedil}

Piribedil was investigated recently in a double-blind placebocontrolled cross-over study $(n=56)$. Results showed that piribedil was not superior to placebo; however, a post-hoc analysis suggested that a subgroup of patients with specific findings in electrocochleography may benefit from piribedil [44].

\section{Other drugs}

a) Misoprostol: is a synthetic prostaglandin E1 analogue which is primarily used to prevent gastric ulcers induced by non-steroidal anti-inflammatory drugs [45]. In a small, placebocontrolled cross-over study, tinnitus severity improved in 33\% of subjects during misoprostol treatment (escalating to 800 $\mathrm{mg}$ /day), while none improved with placebo [46]. A subsequent double-blind placebo-controlled study $(n=40)$ has shown a significant reduction of tinnitus loudness with misoprostol treatment, but no differences in subjective measures of tinnitus severity [47]. A further study has shown efficacy of misoprostol in the treatment for chronic tinnitus in hypertensive and/or diabetic patients [48].

b) Cyclandelate, a vasodilator used in the treatment of cerebrovascular and peripheral vascular disorders, that is believed to act by blocking calcium influx [49], has been investigated for the treatment of tinnitus based on the assumption that some forms of tinnitus may arise from cerebrovascular insufficiency. In an open multicentric clinical trial of patients with tinnitus, vertigo, and visual disturbances, 90-day treatment with cyclandelate reduced the severity and frequency of these symptoms with minimal side effects [50]. However, in a subsequent placebo-controlled double-blind study, cyclandelate did not significantly change audiometric measures of tinnitus loudness and pitch and caused side effects in many patients [51].

c) Furosemide is a loop inhibiting diuretic used to treat congestive heart failure and edema; it inhibits the Na-K-2Cl cotransporter [52], which is expressed in the inner ear as well as in the brain [53]. Furosemide has been proposed as a treatment for tinnitus of cochlear origin because it strongly suppresses the endolymphatic potential and other cochlear responses [54]. In one study, approximately $50 \%$ of patients reported a reduction of tinnitus symptoms following intravenous furosemide treatment. Furosemide has also been found to suppress tinnitus in approximately $40 \%$ of patients with Meniere's disease [55]. In contrast to these positive findings, high doses of furosemide have also been found to induce temporary hearing loss and tinnitus [56].

d) Nimodipine, a calcium antagonist, which crosses the blood - brain barrier and blocks L-type calcium channels [57], is primarily used in the treatment of subarachnoid hemorrhage. Pilot studies also suggest beneficial effects in mood disorders [58]. In animal studies nimodipine significantly reduced tinnitus related behavior caused by high doses of quinine or sodium salicylate [59]. The first open clinical trial suggested positive effects of nimodipine on tinnitus in some patients [60]. However, these results could not be confirmed in a second open clinical trial [61].

e) Cyclobenzaprine a centrally acting muscle relaxant with pharmacological properties similar to amitriptyline, used in the management of fibromyalgia, low back pain, neck pain and temporomandibular disorders. Two open preliminary studies evaluated the action of cyclobenzaprine in the treatment of tinnitus have shown evidences that this drug presents a clear action in reducing tinnitus magnitude as well as in tinnitus discomfort $[62,63]$. Another study conducted in animal model found that cyclobenzaprine was effective in the transient suppression of noise-induced tinnitus in rats. Cyclobenzaprine is a promising drug to treat tinnitus and preliminary results need to be validated in controlled clinical trials [64].

f) Other drugs that have been tested with either limited efficacy or are in need of further controlled trials include the HMG-CoA reductase atorvastatin [65], betahistine [66], Deanxit [67], oxytocin [68], naltrexone [69], ondansetron [70], the phosphodiesterase inhibitors cilostazol [71]and cannabinoids [72].

\section{Drugs in the pipeline for patients with tinnitus}

a) Neramexane: acts as a non-competitive, voltage dependent NMDA antagonist and also blocks $\alpha 9 \alpha 10$ nicotinic cholinergic receptors which are expressed on hair cells in the inner ear [73]. After obtaining positive results from a Phase II trial. (http://clinicaltrials.gov/ct2/show/NCT00405886), Merz Pharmaceuticals is currently conducting Phase III multicenter clinical trials to determine the efficacy, safety and tolerability of Neramexane for treating tinnitus.

b) LidoPAIN TV: is a non-sterile patch delivering lidocaine, which is applied to the periauricular skin region. According to the company's web page, it demonstrated efficacy in a clinical proof of- concept study and has been in phase II trials for tinnitus [15].

c) SPI-1005: contains ebselen which acts as an antioxidant by stimulating glutathione peroxidase. It has completed a phase I trial and phase II trials are planned for the treatment of noiseinduced hearing loss and tinnitus [9].

d) AM 101: is an NMDA antagonist, which is applied topically to the cochlea by round window Injection for the treatment of acute tinnitus. In a randomized, double-blind placebocontrolled German Phase I/II trial in 24 patients with acute tinnitus following noise trauma or sudden deafness, single-dose AM-101 intratympanic injection was well tolerated. It also had a positive effect on tinnitus loudness. Currently a double-blind, randomized, placebo-controlled Phase II trial with cochlear application of AM-101 is being carried out. The study involves patients with acute $(<3$ months) noise-induced tinnitus that 
have not responded to glucocorticoid treatment. The same company has also a second compound under development (AM-102). This compound is of unidentified pharmacologic activity and is also delivered by intratympanic application. It is currently in preclinical test [9].

e) Vestipitant: is a novel antagonist of the neurokinin-1 (NK-1) receptor which binds substance P. Neurokinin receptors are present in the inner ear and therefore represent a potential therapeutic target for tinnitus [74]. Vestipitant and the combination of vestipitant and paroxetine are currently undergoing a phase II clinical trial for the treatment of tinnitus [75].

f) EGb-761: is a concentrated extract of Ginkgo biloba, enriched in flavonoids and terpenes, which has a broad spectrum of pharmacologic actions, including a free-radical scavenger effect and which has shown efficacy for tinnitus in a phase I trial (Table 1).

Table 1: Drugs under development for tinnitus.

\begin{tabular}{|c|c|c|c|}
\hline Compound & Indications & Stage of Development & Mechanism of Action \\
\hline Neramexane & Tinnitus, Alzheimer's and pain & Phase III & $\begin{array}{l}\text { NMDA antagonist, block } \alpha 9 \alpha 10 \text { nicotinic } \\
\text { cholinergic receptors }\end{array}$ \\
\hline LidoPAIN TV & Tinnitus and vertigo & Phase II & $\begin{array}{l}\text { Local anesthesia of preauricular nerve } \\
\text { ending }\end{array}$ \\
\hline SPI-1005 & $\begin{array}{l}\text { Prevention and treatment of noise induced } \\
\text { hearing loss and tinnitus }\end{array}$ & Phase II & Antioxidant \\
\hline AM 101 & Tinnitus and hearing loss & Phase II & NMDA antagonist \\
\hline AM-102 & Tinnitus and hearing loss & Preclinical & Unidentified mechanism of action \\
\hline Vestipitant & Depression, tinnitus and anxiety & Phase II & $\begin{array}{l}\text { Neurokinin -1receptor antagonist and } \\
\text { serotonin re- uptake inhibitor }\end{array}$ \\
\hline EGb-761 & $\begin{array}{l}\text { Cognitive disorders, Alzheimer, vertigo and } \\
\text { tinnitus }\end{array}$ & Phase I & Free radical scavenger \\
\hline
\end{tabular}

\section{Conclusion}

The use of drugs to treat tinnitus is widespread. Some subjects report benefits from drugs, while others do not. There is likely a placebo effect influencing those results. It is also noteworthy that many patients develop side effects. Patients using drugs might not be aware of the side effects, even though warnings might be present on labels. Tyler et al. [76] stated that there are many subgroups of tinnitus and it might be that different drugs help some subtypes tinnitus sufferers, but not all tinnitus sufferers. Despite the huge potential market, we still lack approved pharmacological treatments for tinnitus. Despite the significant unmet clinical need for a safe and effective drug targeting tinnitus relief, there is currently no single FDA approved drug on the market. The empirical approach, in combination with new developments in information technology such as the advent of big data approaches in medicine, might facilitate the identification of promising drugs. We have also stressed the importance of examining individual results.

\section{References}

1. Stouffer JL, Tyler RS (1990) Characterization of tinnitus by tinnitus patients. Journal of Speech and Hearing Disorders 55(3): 439-453.

2. Henry JA, Dennis KC, Schechter MA (2005) General review of tinnitus: prevalence, mechanisms, effects, and management. Journal of speech, language, and hearing research 48(5): 1204-1235.

3. Tyler R, Ji H, Perreau H, Witt S, Noble W, et al. (2014) Development and validation of the Tinnitus Primary Function Questionnaire. American Journal of Audiology 23(3): 260-272.

4. Tyler RS (2006a) Neurophysiological models, psychological models, and treatments for tinnitus. In RS Tyler (Ed.), Tinnitus treatment: Clinical protocols Thieme, New York, NY, pp. 1-22.
5. Dauman R, Tyler RS (1992) Some considerations on the classification of tinnitus. In JM Aran, R Dauman (Eds.), Proceedings of the fourth International Tinnitus Seminar. Amsterdam, The Netherlands: Kugler Publications, pp. 225-229.

6. Tyler RS (2012) Patient preferences and willingness to pay for tinnitus treatment. Journal of the American Academy of Audiology 23(2): 115125 .

7. Baguley DM, Jones S, Wilkins I, Axon PR, Moffat DA (2005) The inhibitory effect of intravenous lidocaine infusion on tinnitus after trans labyrinthine removal of vestibular schwannoma: a double-blind, placebo-controlled, crossover study. Otol Neurotol 26(2): 169-176.

8. Nagler SM (2003) Tinnitus: A patient's perspective. Otolaryngol Clin North Am 36: 235-238.

9. Langguth B, Salvi R, Elgoyhen AB (2009) Emerging pharmacotherapy of tinnitus. Expert Opin Emerg Drugs 14(4): 687-702.

10. Barany R (1935) Influencing the buzz through intravenously injected local anesthetics. Acta Otolarngologica 23: 201-203.

11. Trellakis S, Benzenberg D, Urban BW, Friederich P (2006) Differential lidocaine sensitivity of human voltage-gated potassium channels relevant to the auditory system. Otol Neurotol 27(1): 117-123.

12. Lenkowski PW, Shah BS, Dinn AE, Lee K, Patel MK (2003) Lidocaine block of neonatal Nav1.3 is differentially modulated by co-expression of beta1 and beta3 subunits. Eur J Pharmacol 467(1-3): 23-30.

13. Josephson IR (1988) Lidocaine blocks $\mathrm{Na}$, Ca and K currents of chick ventricular myocytes. J Mol Cell Cardiol 20(7): 593-604.

14. Lenarz T (1986) Treatment of tinnitus with lidocaine and tocainide. Scand Audiol 26: 49-51.

15. Santosh Kumar Swain, Saumyadarshan Nayak, Jayprakash Russel Ravan, Mahesh Chandra Sah (2016) Tinnitus and its current treatment-Still an enigma in medicine. Journal of the Formosan Medical Association 115(3): 139-144 
16. Moller AR (2000) Similarities between severe tinnitus and chronic pain. J Am Acad Audiol 11: 115-124.

17. Gross G, Xin X, Gastpar M (1991) Trimipramine: pharmacological reevaluation and comparison with clozapine. Neuropharmacology 30(11): 1159-1166.

18. Sullivan MD, RA Dobie, CS Sakai et al (1989) Treatment of depressed tinnitus patients with nortriptyline. Ann oto Rhinol Laryngol 98: 867872.

19. Sullivan M, W Katon, J Russo et al (1993) A randomized trial of nortriptyline for severe chronic tinnitus. Effects on depression, disability, and tinnitus symptoms. Arch Intern Med 153: 2251-2259.

20. Katon W, M Sullivan, J Russo, R Dobie, C Sakai (1993) Depressive symptoms and measures of disability: a prospective study. J Affect Disord 27: 245-254.

21. Bayar N, B Boke, E Turan, E Belgin (2001) Efficacy of amitriptyline in the treatment of subjective tinnitus. J Otolaryngol 30: 300-303.

22. Podoshin L, Y Ben David, M Fradis, S Malatskey, H Hafner (1995) Idiopathic subjective tinnitus treated by amitriptyline hydrochloride/ biofeedback. Int. Tinnitus J 1: 54-60.

23. Mihail RC, JM Crowley, BE Walden et al (1988) The tricyclic trimipramine in the treatment of subjective tinnitus. Ann Otol Rhinol Laryngol 97 120-123.

24. Zoger S, J Svedlund and KM Holgers (2006) The effects of sertraline on severe tinnitus suffering--a randomized, double- blind, placebocontrolled study. J Clin Psychopharmacol 26: 32-39.

25. Robinson S (2007) Antidepressants for treatment of tinnitus. Prog Brain Res 166: 263-271.

26. Eggermont JJ, LE Roberts (2004) The neuroscience of tinnitus. Trends Neurosci 27: 676-682.

27. Johnson RM, R Brummett and A Schleuning (1993) Use of alprazolam for relief of tinnitus. A double-blind study. Arch Otolaryngol Head Neck Surg 119: 842-845.

28. Bahmad FM Jr, AR Venosa and CA Oliveira (2006) Benzodiazepines and GABAergics in treating severe disabling tinnitus of predominantly cochlear origin. Int. Tinnitus J 12: 140-144.

29. Busto U, L Fornazzari and CA Naranjo (1988) Protracted tinnitus after discontinuation of long-term therapeutic use of benzodiazepines. J Clin Psychopharmacol 8: 359-362.

30. Ettinger $A B$ and CE Argoff (2007) Use of antiepileptic drugs for nonepileptic conditions: psychiatric disorders and chronic pain. Neuro therapeutics 4: 75-83.

31. Neligan A, SD Shorvon (2009) The history of status epilepticus and its treatment. Epilepsia 50 Suppl 3: 56-68.

32. Catterall WA (1999) Molecular properties of brain sodium channels: an important target for anticonvulsant drugs. Adv Neurol 79: 441-456.

33. Sanchez TG, AP Balbani, RS Bittar et al (1999) Lidocaine test in patients with tinnitus: rationale of accomplishment and relation to the treatment with carbamazepine. Auris Nasus Larynx 26: 411-417.

34. Levine RA (2006) Typewriter tinnitus: a carbamazepine responsive syndrome related to auditory nerve vascular compression. ORL J Otorhinolaryngol Relat Spec 68: 43-47.

35. Levendoglu F, CO Ogun, O Ozerbil, TC Oqun, H Uqurlu (2004) Gabapentin is a first line drug for the treatment of neuropathic pain in spinal cord injury. Spine 29: 743-751.

36. Bauer CA, TJ Brozoski (2006) Effect of gabapentin on the sensation and impact of tinnitus. Laryngoscope 116: 675-681.

37. Witsell DL, MT Hannley, S Stinnet, DL Tucci (2007) Treatment of tinnitus with gabapentin: a pilot study. Otol Neurotol 28: 11-15.
38. Eggermont JJ (2007) Pathophysiology of tinnitus. Prog Brain Res 166: $19-35$

39. Ehrenberger K, Felix D (1995) Receptor pharmacological models for inner ear therapies with emphasis on glutamate receptors: a survey. Acta Otolaryngol 115: 236-40.

40. Denk DM, Heinzl H, Franz P, Ehrenberger K (1997) Caroverine in tinnitus treatment. A placebo-controlled blind study. Acta Otolaryngol 17(6): 825-830.

41. Azevedo AA, RR Figueiredo (2007) Treatment of tinnitus with acamprosate. Prog Brain Res 166: 273-277.

42. Memo M, Battaini F, Spano PF, Trabucchi M (1981) Sulpiride and the role of dopaminergic receptor blockade in the antipsychotic activity of neuroleptics. Acta Psychiatr Scand 63(4): 314-324.

43. Lopez Gonzalez MA, Santiago AM, Esteban Ortega F (2007) Sulpiride and melatonin decrease tinnitus perception modulating the audito limbic dopaminergic pathway. J Otolaryngol 36(4): 213-219.

44. Azevedo AA, Langguth B, de Oliveira PM, Figueiredo RR (2009) Tinnitus treatment with piribedil guided by electrocochleography and acoustic oto emissions. Otol Neurotol 30(5): 676-680.

45. Lazzaroni M and GB Porro (2009) Management of NSAIDinduced gastrointestinal toxicity: focus on proton pump inhibitors. Drugs 69: $51-$ 69.

46. Briner W, J House and M OLeary (1993) Synthetic prostaglandin E1 misoprostol as a treatment for tinnitus. Arch Otolaryngol Head Neck Surg 119: 652-654

47. Yilmaz I, B Akkuzu, O Cakmak, LN Ozluoglu (2004) Misoprostol in the treatment of tinnitus: a double-blind study. Otolaryngol Head Neck Surg 130: 604-610

48. Akkuzu B, I Yilmaz, O Cakmak et al (2004) Efficacy of misoprostol in the treatment of tinnitus in patients with diabetes and/or hypertension. Auris Nasus Larynx 31: 226-232.

49. Timmerman H (1987) Calcium modulation and clinical effect. Profile of cyclandelate. Drugs 33 (Suppl 2): 1-4

50. Memin Y (1987) Perceived efficacy of cyclandelate in the treatment of cochleovestibular and retinal disturbances related to cerebrovascular insufficiency. A study in general practice comprising 2772 patients. Drugs 33(Suppl 2): 120-124.

51. Hester TO, G Theilman, W Green et al (1998) Cyclandelate in the management of tinnitus: a randomized, placebocontrolled study. Otolaryngol Head Neck Surg 118: 329-332.

52. Shiozaki A, Miyazaki H, Niisato N, Nakahari T, Iwasaki Y, et al. (2006) Furosemide, a blocker of $\mathrm{Na}+/ \mathrm{K}+/ 2 \mathrm{Cl}-$ cotransporter, diminishes proliferation of poorly differentiated human gastric cancer cells by affecting G0/G1 state. J Physiol Sci 56(6): 401-406.

53. Chen H, Sun D (2005) The role of Na-K-Cl co-transporter in cerebral ischemia. Neurol Res 27(3): 280-286

54. Rybak LP, Morizono T (1982) Effect of furosemide upon endolymph potassium concentration. Hear Res 7(2): 223-231.

55. Futaki T, Kitahara M, Morimoto M (1977) A comparison of the furosemide and glycerol tests for Meniere's disease. With special reference to the bilateral lesion. Acta Otolaryngol 83(3-4): 272-278.

56. Ho KM, Sheridan DJ (2006) Meta-analysis of frusemide to prevent or treat acute renal failure. BMJ 333(7565): 420.

57. Scriabine A, van den KW (1988) Pharmacology of nimodipine. A review. Ann N Y Acad Sci 522: 698-706.

58. Goodnick PJ (1995) Nimodipine treatment of rapid cycling bipolar disorder. J Clin Psychiatry 56(7): 330

59. Jastreboff PJ, Brennan JF, Sasaki CT (1991) Quinine-induced tinnitus in rats. Arch Otolaryngol Head Neck Surg 117(10): 1162-1166. 
60. Theopold, HM (1985) Nimodipine (Bay e 9736) a new therapy concept in diseases of the inner ear. Laryngol Rhinol Otol. Stuttg 64(12): 609613.

61. Davies E, Knox E, Donaldson I (1994) The usefulness of nimodipine, an L-calcium channel antagonist, in the treatment of tinnitus. Br J Audiol 28(3): 125-129.

62. Coelho C, Figueiredo R, Frank E, Burger J, Schecklmann M, et al. (2012) Reduction of Tinnitus Severity by the Centrally Acting Muscle Relaxant Cyclobenzaprine: An Open-Label Pilot Study. Audiology and Neurotology 17(3): 179-188.

63. Vanneste S, Figueiredo R, Ridder D (2012) Treatment of tinnitus with cyclobenzaprine: an open-label study. Int Journal of Clinical Pharmacology and Therapeutics 50(5): 338-344.

64. Lobarinas E, Blair C, Spankovich C, Le Prell C (2014) Partial to Complete Suppression of Unilateral Noise-Induced Tinnitus in Rats after Cyclobenzaprine Treatment. Journal of The Association for Research in Otolaryngology 16(2): 263-272.

65. Hameed MK, Sheikh ZA, Ahmed A, Najam A (2014) Atorvastatin in the management of tinnitus with hyperlipidemias. J Coll Phys Surg Pak 24: 927-930.

66. Sonmez O, Kulahli I, Vural A, Sahin MI, Aydin M (2013) The evaluation of ozone and betahistine in the treatment of tinnitus. Eur Arch Otorhinolaryngol 270: 1999-2006.

67. Meeus O, De Ridder D, Van de Heyning P (2011) Administration of the combination clonazepam-Deanxit as treatment for tinnitus. Otol Neurotol 32: 701-709.

68. Azevedo AA, Figueiredo RR, Elgoyhen AB, Langguth B, Penido NO, et al. (2017) Tinnitus treatment with oxytocin: a pilot study. Front Neurol 8: 494.
69. Vanneste S, Azevedo A, De Ridder D (2013) The effect of naltrexone on the perception and distress in tinnitus: an open-label pilot study. Int J Clin Pharmacol Ther 51: 5-11.

70. Taslimi S, Vahidi H, Pourvaziri A, Modabbernia A, Fallah AY, et al. (2013) Ondansetron in patients with tinnitus: randomized double-blind placebo-controlled study. Eur Arch Otorhinolaryngol 270: 1635-1641.

71. Lim HW, Kim TS, Kang WS, Song CI, Baek S, Chung JW (2016) Effect of a 4-week treatment with cilostazol in patients with chronic tinnitus: a randomized, prospective, placebo-controlled, double-blind, pilot study. J Int Adv Otol 12: 170-176.

72. Smith PF, Zheng Y (2016) Cannabinoids, cannabinoid receptors and tinnitus. Hear Res 332: 210-216.

73. Plazas PV, Savino J, Kracun S, Gomez Casati ME, Katz E, Parsons CG, et al. (2007) Inhibition of thealpha9alpha10 nicotinic cholinergic receptor by neramexane, an open channel blocker of N-methyl-D-aspartate receptors. Euro J Pharmacol 566(1-3): 11-19.

74. Sun W, Ding DL, Wang P, Sun J, Jin X, Salvi RJ (2004) Substance P inhibits potassium and calcium currents in inner ear spiral ganglion neurons. Brain Res 1012(1-2): 82-92.

75. Berthold Langguth, Ana Belen Elgoyhen, Christopher R Ceder Roth (2019) Therapeutic Approaches to the Treatment of Tinnitus. Annual Review of Pharmacology and Toxicology 59: 291-313.

76. Tyler R, Coelho C, Tao P, Ji H, Noble W, et al. (2008) Identifying tinnitus subgroups with cluster analysis. Am J Audiol 17(2): 176-184.
This work is licensed under Creative Commons Attribution 4.0 License

To Submit Your Article Click Here: Submit Article

DOI: $10.32474 /$ SJO.2019.02.000147

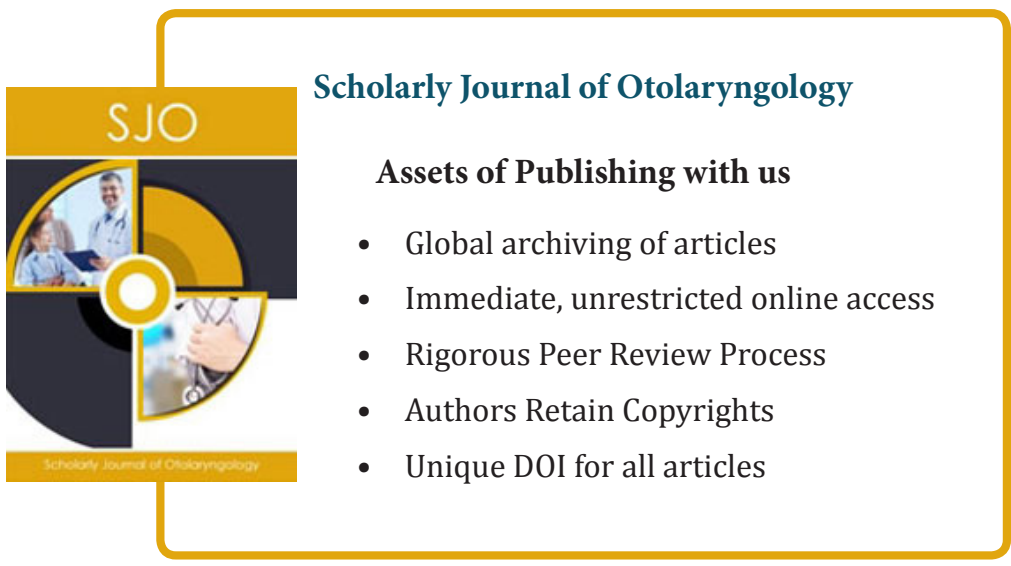

